\title{
Desenvolvimento e avaliação das propriedades psicométricas da versão brasileira do Addiction Severity Index 6 (ASI-6) Light
}

\author{
Development and evaluation of psychometric properties of the \\ Brazilian version of Addiction Severity Index 6 (ASI-6) Light
}

Lídia Reis Fernandes', Fernando Antonio Basile Colugnati², Laisa Marcorela Andreoli Sartes

\section{RESUMO}

Objetivo: Desenvolver e avaliar as propriedades psicométricas de uma versão brasileira reduzida do Addiction Severity Index 6 Light (ASI- 6 Light) previamente proposta com base em um estudo de validação dos construtos do instrumento e desenvolver os novos escores de cada área do instrumento baseados na Teoria de Resposta ao Item (TRI). Métodos: Foram entrevistados 200 sujeitos, 100 com uso problemático de álcool e outras drogas e 100 sem uso problemático. Foram calculados os escores dos indivíduos com base na TRI. As propriedades psicométricas foram avaliadas pela correlação entre os escores do ASI-6 Light e do Alcohol, Smoking and Substance Involvement Screening Test (ASSIST), padrão-ouro do estudo. Foram avaliados os índices de sensibilidade e especificidade. Resultados: Foi encontrada alta correlação entre os escores da área "álcool" do ASI-6 Light e os escores do ASSIST em relação ao álcool ( $r=0,79)$, correlações moderadas em relação ao tabaco $(r=0,47)$ e cocaína/crack $(r=0,44)$ e baixa $(r=0,39)$ em relação à maconha. Ao correlacionarem-se os escores do ASSIST e os escores da área "drogas" do ASI-6 Light, obteve-se alta correlação em relação à cocaína/ $\operatorname{crack}(r=0,85)$, correlações moderadas em relação ao tabaco $(r=0,57)$ e maconha $(r=0,68)$ e baixa $(r=0,29)$ em relação ao álcool. A área sob a curva ROC da área "álcool" foi de 0,93 e a da área "drogas" foi de 0,88. Conclusão: Boas evidências de validade das áreas "álcool" e "drogas" foram apresentadas. Essa nova versão tornou-se um instrumento de fácil manejo e de rápida aplicação, contendo os itens que melhor avaliam a gravidade de problemas.

\section{Palavras-chave \\ Estudos de validação, Addiction Severity Index, substâncias psicotrópicas.}

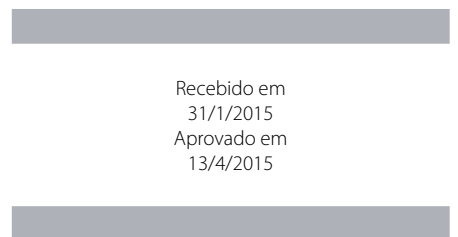

DOI: $10.1590 / 0047-2085000000068$

\section{ABSTRACT}

Objective: To develop and evaluate the psychometric properties of a shortened Brazilian version of the Addiction Severity Index 6 (ASI-6 Light), previously proposed from a validation study of the instrument constructs and developing new scores for each instrument area based on the Item Response Theory (IRT). Methods: Two hundred subjects were interviewed, 100 with the abuse of alcohol and other drugs and 100 without problematic use. The scores of individuals were calculated from the Item Response Theory. The psychometric properties were evaluated by the correlation between the scores of the ASI-6 Light and Alcohol, Smoking and Substance Involvement Screening Test (ASSIST), the gold standard study. The sensitivity and specificity rates were calculated. Results: A high correlation between

1 Universidade Federal de Juiz de Fora (UFJF), Departamento de Psicologia, Centro de Referência em Pesquisa, Intervenção e Avaliação em Álcool e Outras Drogas. 2 UFJF, Faculdade de Medicina.

Endereço para correspondência: Laisa Marcorela Andreoli Sartes

Campus Universitário, Instituto de Ciências Humanas

Rua José Lourenço Kelmer, s/n

E-mails: laisa.sartes@gmail.com; laisa.sartes@uff.edu.br 


\section{Keywords}

Validation study, Addiction Severity Index, psychotropic substances. the scores of the area "alcohol" ASI-6 Light and scores ASSIST regarding alcohol was found $(r=0.79)$, moderate correlations with tobacco $(r=0.47)$ and cocaine/crack $(r=0.44)$ and low $(r=0.39)$ with respect to marijuana. Correlating ASSIST scores and the scores of the area "drugs" ASI-6 Light obtained a high correlation to cocaine/crack $(r=0.85)$, moderate correlations with tobacco $(r=0.57)$ and marijuana $(r=0.68)$ and low $(r=0.29)$ compared to alcohol. The area under the ROC curve of area "alcohol" was 0.93 and the area "drugs" was 0.88. Conclusion: Good evidence of validity of the area "alcohol" and "drugs" were presented. This new version has become an instrument for easy handling and quick application, containing the items that best assess the severity of problems.

\section{INTRODUÇÃO}

No Brasil e outros países, o consumo de substâncias psicotrópicas gera sérias consequências para os indivíduos que as consomem, suas famílias e para a comunidade em geral, o que o torna um relevante problema para a saúde pública'. Com isso, vários estudos são realizados para contribuir com programas de prevenção e tratamento.

A avaliação do fenômeno e as intervenções nele realizadas, idealmente, devem estar de acordo com a metodologia científica. Há necessidade de uma linguagem comum entre os pesquisadores que trabalham na área, o que contribuiu para o desenvolvimento de instrumentos padronizados, entre eles, as escalas de avaliação. Uma análise adequada e abrangente é essencial tanto para o desenvolvimento de um tratamento apropriado quanto para a realização de estudos sobre a efetividade das intervenções propostas e problemas associados ao consumo de álcool e outras drogas².

O Addiction Severity Index (ASI) é um instrumento que permite colher informações sobre vários aspectos da vida de pessoas com problemas associados ao abuso e à dependência de drogas, como questões médicas, situação ocupacional, aspectos legais, sociofamiliares, psiquiátricos, além do uso de álcool e de outras drogas. Foi desenvolvido em 1979 por A. Thomas McLellan e colegas do Center for Studies of Addiction, na Filadélfia, USA, inicialmente para propósitos de pesquisa ${ }^{3}$. É amplamente utilizado e já foi validado para diferentes línguas e contextos ${ }^{4-9}$. Trata-se de uma entrevista semiestruturada, com duração de 45 a 90 minutos, que deve ser realizada por entrevistadores treinados. É dividida nas sete áreas de avaliação acima citadas, que são formadas por itens objetivos sobre o número, a intensidade e a duração dos sintomas ocorridos durante toda a vida do indivíduo e nos últimos 30 dias que antecedem a entrevista. O ASI estima a gravidade do problema em cada área, sendo, assim, um instrumento útil para avaliação clínica e em pesquisa. Em virtude dessas vantagens, pode ser utilizado também em estudos longitudinais sobre o resultado do tratamento ${ }^{10}$.

Atualmente, o ASI encontra-se na sexta versão. Um estudo transversal e multicêntrico foi realizado em quatro capitais de estados brasileiros a fim de testar as propriedades psicométricas do ASI-6. As correlações entre os escores das áreas "álcool" e "drogas" do ASI-6 e o instrumento concorrente (Alcohol, Smoking and Substance Involving Screening Test ASSIST) ${ }^{11}$ foram altas $(0,72$ e 0,89, respectivamente). $O$ alfa de Cronbach para as subescalas do ASI-6 variou de 0,64 a 0,95. A maioria das áreas do ASI-6 mostrou alta correlação entre as diferentes medidas no teste-reteste. Este estudo apontou as boas propriedades psicométricas de confiabilidade e validade do ASI-6 para a cultura brasileira ${ }^{12}$.

Porém, duas questões vêm sendo levantadas quanto à utilização do instrumento. Por um lado, a aplicação do ASI requer disponibilidade de tempo dos profissionais, nem sempre encontrado em serviços de saúde. Por outro, sua utilização em pesquisa vinha sendo questionada devido ao uso de escores de gravidade subjetivos, que conferem um enviesamento de resultados ${ }^{13}$. Além disso, são raros os estudos que utilizam análises específicas para avaliação dos construtos e dos itens ${ }^{14}$. Nesse sentido, foi realizado um estudo no Brasil para avaliar as propriedades psicométricas das sete áreas do ASI-6, com foco na avaliação de construto e dos itens, utilizando uma combinação de análises da Teoria de Resposta ao Item (TRI) e Análise Fatorial (AF). Foi proposta uma seleção de itens para uma versão reduzida do ASI-6 brasileiro que apresentaram melhores propriedades de discriminação e dificuldade de acordo com a TRl, com exceção da área "emprego/ sustento", cuja análise pela AF se mostrou mais adequada. Dessa forma, os construtos das sete áreas do ASI-6 poderiam ser avaliados de maneira consistente utilizando apenas 96 das 252 questões originais (344 itens), gerando uma versão mais breve e otimizada do ASI-6 - o ASI-6 Light $^{15}$.

O ASI-6 Light poderia reduzir o tempo necessário para sua aplicação, melhorar a qualidade dos dados, por reduzir o cansaço do paciente decorrente de uma longa aplicação, e fornecer escores para pesquisa que melhor avaliam os construtos - a gravidade de problemas em cada área. Diante desse contexto, o presente estudo teve como objetivo desenvolver e avaliar as propriedades psicométricas de uma versão brasileira reduzida do ASI-6 Light, proposta com base em um estudo de validação dos construtos do instrumento. Especificamente, buscou-se desenvolver os novos escores de cada área do instrumento baseados na TRI. 


\section{MÉTODOS}

Trata-se de um estudo transversal, de natureza quantitativa, que utilizou dados coletados por meio de entrevistas a 200 sujeitos, usuários ou não de álcool e outras drogas, que utilizavam os serviços de saúde e de acolhimento social da cidade de Juiz de Fora, MG. As entrevistas foram realizadas por entrevistadores da área de psicologia, devidamente treinados para aplicação dos instrumentos, em local isolado, estando presentes somente o entrevistador e o entrevistado. Foram utilizados um questionário de dados sociodemográficos, o ASSIST e o ASI-6 Light.

O ASI-6 Light é composto por 96 questões, distribuídas em sete áreas ("álcool", "drogas", "sociofamiliar", "legal", "psiquiátrica", "emprego" e "saúde") 12,15,16. Um estudo-piloto realizado com 17 indivíduos que faziam uso problemático de substâncias (indicado pelo ASSIST) permitiu avaliar a adequação do novo conjunto de questões para a realização da entrevista com o paciente, bem como fazer algumas alterações. A primeira foi o desmembramento de itens para cocaína e crack.

$\mathrm{Na}$ versão original era utilizada a classe da cocaína para todas as perguntas. No entanto, os entrevistados respondiam e compreendiam as duas formas de utilização como se fossem drogas diferentes. Na área psiquiátrica, foi identificado que não havia uma sequência lógica do que estava sendo questionado. Alguns itens se referiam a sintomas ocorridos nos últimos 30 dias, outros, à ocorrência na vida, enquanto alguns se referiam aos dois períodos.

Visando permitir a lógica de raciocínio aos entrevistados, foi necessário manter todos os itens com questões relacionadas tanto à ocorrência de sintomas na vida como durante os últimos 30 dias. No entanto, somente os itens que obtiveram boas propriedades psicométricas no estudo de Sartes ${ }^{15}$ entram no cálculo do escore da área. Em média, 17,7 minutos foram necessários para aplicar o ASI-6 Light, enquanto para a aplicação do ASI-6 tradicional são necessários entre 45 e 90 minutos.

O ASSIST ${ }^{11}$ é composto por oito questões sobre o uso de álcool e outras drogas nos últimos três meses. Fornece o padrão de uso de 13 classes de substâncias, classificando em baixo risco, uso de risco e indicativo de dependência. Os resultados de um estudo, cujo objetivo foi avaliar as propriedades psicométricas do ASSIST, demostraram que tal instrumento é capaz de obter informações precisas relativas ao uso de substâncias psicoativas e o nível de risco associado ao uso de substâncias ${ }^{17}$. No geral, o ASSIST mostra boa validade concorrente, de construto e discriminativa, podendo, então, classificar adequadamente o padrão de uso em três níveis de gravidade, conforme descrito anteriormente.

Com base na aplicação do ASSIST, os sujeitos foram classificados segundo dois grupos. O grupo 1 foi composto por 100 indivíduos que apresentavam uso problemático de álcool e outras drogas. Esses indivíduos deveriam atingir pontuação acima de 10 para álcool e acima de 4 para outras drogas (exceto tabaco). O grupo 2 foi formado pelos 100 indivíduos que não apresentavam consumo problemático de álcool e outras drogas e atingiam pontuação máxima de 3 no ASSIST. Dessa forma, o ASSIST se constituiu como critério (padrão-ouro) das áreas de uso de álcool e drogas do ASI-6 Light nas análises de propriedades diagnósticas e validade concorrente.

O estudo foi aprovado pelo Comitê de Ética em Pesquisa da Universidade Federal de Juiz de Fora (CEP/UFJF, protocolo 417.119). Cada sujeito que concordou em participar da pesquisa assinou o Termo de Consentimento Livre e Esclarecido sobre sua participação em pesquisas.

Os dados foram transferidos para um banco de dados por dois digitadores e foi realizada uma análise crítica dos dados por meio da digitação cruzada. Análises descritivas foram conduzidas para as variáveis sociodemográficas, padrão de consumo de substâncias fornecido pelo ASSIST, e para os escores de seis áreas do ASI-6 Light (exceto emprego). Para comparação dos grupos, foram utilizados o teste do Chi-quadrado para as variáveis nominais, teste t de Student para variáveis numéricas e o teste de Mann-Whitney para variáveis ordinais.

As propriedades psicométricas do novo instrumento foram avaliadas por meio da validade concorrente e do cálculo do equilíbrio entre sensibilidade e especificidade. Para a validade concorrente, foram realizadas análises de correlação de Pearson entre as áreas "álcool" e "drogas" do ASI-6 Light e os escores do ASSIST utilizados como padrão-ouro. A análise de curva ROC (Received Operator Characteristic) avaliou a Área Sob a Curva (ASC) e estimou as propriedades de sensibilidade e especificidade do instrumento.

Os escores do ASI-6 Light dos indivíduos foram gerados com base na TRI, com exceção da área "emprego e sustento", que não obteve boa avaliação das propriedades psicométricas segundo a TRI no estudo de Sartes ${ }^{15}$. Para isso, os itens politômicos de $k$ categorias (que assume valor 1 se respondeu à categoria e 0 caso contrário) foram transformados em variáveis $k$ indicadoras, para cada uma delas. Os coeficientes para esses itens, gerados na calibração com base no Modelo de Resposta Gradual (GRM) ${ }^{18}$, foram montados na respectiva ordem de cada categoria. Os escores do instrumento ASI, em suas seis dimensões, foram estimados por meio do método de Máxima Verossimilhança' ${ }^{19}$, com a função thetaEst do pacote CatR do software estatístico R ${ }^{20}$.

Antes de serem submetidas ao cálculo dos escores dos indivíduos, foi necessário o ajuste ou recategorização de alguns itens. As respostas às questões $\mathrm{P} 1$ a $\mathrm{P} 7$, relativas a sintomas da área "psiquiátrica", foram recategorizadas para que ficassem a favor do construto em termos de gravidade de problemas psiquiátricos. Assim, as respostas originais $0=$ não, $1=\operatorname{sim}, 2=\operatorname{sim}$, mas sob efeito de drogas, foram alteradas 
para 0 = não, $1=$ sim, sob efeito de drogas, $2=$ sim; pois, do ponto de vista psiquiátrico, ter apresentado o sintoma sem o efeito da droga é mais grave do que o ter tido sob efeito da droga ou sob efeito de abstinência. Foram alteradas, ainda, as variáveis dos itens com respostas do tipo não se aplica. A falta de resposta ocorreu somente nas áreas "drogas" e "sociofamiliar", entre as questões D13 e D22; F6 e F16. Se o entrevistado respondeu na área "drogas" que não fazia uso de drogas, ou na área "sociofamiliar" que não tinha filhos ou não morava com nenhuma criança, as perguntas seguintes foram saltadas. Para o cálculo dos escores desses indivíduos, assumiu-se valor 0 para as respostas do tipo não se aplica, sendo esse o código para menor gravidade do problema. A área "psiquiátrica" não continha perguntas com respostas do tipo não se aplica. No entanto, os 17 primeiros entrevistados (estudo-piloto) não responderam a algumas questões, já que elas foram incluídas após o estudo-piloto. As respostas faltantes foram preenchidas com zero no banco de dados. Em ambas as situações o 0 é entendido como o não uso ou nível de menor gravidade. Essa ação corrobora o paradigma da TRI, em que a probabilidade de resposta à maior intensidade nas escalas do tipo Likert aumenta em função da gravidade do entrevistado. Assim, se o entrevistado não é um dependente ou usuário de risco de drogas, o nível é dado como 0.

Visando reduzir o tempo de aplicação e ao mesmo tempo manter as propriedades dos itens, a versão do ASI-6 Light apresentada neste estudo propôs a redefinição das opções de resposta para quatro categorias dos itens que utilizavam escala do tipo Likert de cinco pontos, a saber: $0=$ nada, 1 = levemente, 2 = consideravelmente, 3 = extremamente. Originalmente, existia ainda a opção de resposta "moderadamente". No entanto, no estudo de Sartes ${ }^{15}$ as Curvas Características dos Itens $(\mathrm{CCl})$ com resposta Likert mostraram que as categorias "levemente" e "moderadamente" estavam sobrepostas. Nesses casos, segundo Modelo de Resposta Gradual, as categorias podem ser reagrupadas sem perda das propriedades do item. Para avaliar isso, novas análises baseadas na TRI foram realizadas para avaliação dos parâmetros $a$ (discriminação) e $b$ (dificuldade) dos itens, agora com quatro categorias, utilizando o mesmo procedimento proposto por Sartes ${ }^{15}$. Essas análises foram realizadas no software Parscale ${ }^{21}$. Os novos parâmetros $a$ e $b$ foram, então, utilizados para o cálculo dos escores dos indivíduos no R software livre, conforme descrito anteriormente.

Segundo o paradigma da TRI, os escores foram estimados e posicionados na mesma escala em que os itens são posicionados. A escala do traço latente (construto), definida pela gravidade de problemas em cada área, possui, em termos práticos, oito níveis de gravidade, que variam de -4 a +4 em uma métrica de média $=0$ e desvio-padrão $=1$. Ou seja, os níveis de gravidade foram constituídos por categorias formadas pelos desvios-padrão em relação à média. Com a redução do número de categorias dos itens politômicos de cinco para quatro, o posicionamento dos itens na escala foi refeito, utilizando-se os novos parâmetros $a$ e $b$. Essas novas análises foram realizadas visando verificar se o posicionamento dos itens permaneceria o mesmo, o que ocorreu em todas as áreas, com exceção da área "sociofamiliar", em que alguns itens foram posicionados em níveis diferentes do descrito por Sartes ${ }^{15}$. Dessa forma, a interpretação dos níveis de gravidade, realizada a com base nos itens que os compõem, não foi alterada, com exceção da área "sociofamiliar".

\section{RESULTADOS}

A aplicação do ASI-6 Light durou em média 17,7 minutos (máx. $=44$ e mín. $=9 ; d p=6,5$ ). O tempo variou conforme a gravidade do padrão de consumo medido pelo ASSIST, o número de substâncias utilizadas e os problemas associados. Com relação ao perfil sociodemográfico, o grupo de usuários foi formado por $68 \%$ de homens, com idade média de 38 anos ( \pm 11$)$; $54 \%$ tinham mais de dois anos de defasagem escolar; 86\% não trabalhavam; 43\% eram de religião protestante; $51 \%$ estavam entre as classes socioeconômicas B2 e C1. O grupo de não usuários foi formado por $85 \%$ de mulheres, com idade média de 46 anos ( \pm 13 ); 77\% não tinham defasagem escolar; $57 \%$ não trabalhavam; $54 \%$ eram de religião católica; 59,9\% estavam entre as classes C1 e C2. Foram encontradas diferenças estatísticas em todas as características ( $p<0,0001)$, com exceção da religião $(p=0,512)$. Quanto ao padrão de consumo de drogas nos últimos três meses entre os usuários, as porcentagens obtidas foram: tabaco (67\% - uso de risco; 17\% - dependência); álcool (37\% - uso de risco; 36\% - dependência); maconha (43\% - uso de risco; $12 \%$ - dependência); cocaína/crack (13\% - uso de risco; 49\% - dependência); estimulantes, inalantes, sedativos e alucinógenos (7\% - uso de risco).

A figura 1 apresenta a distribuição de frequência dos indivíduos nos níveis de gravidade com base nos novos escores do ASI-6 Light de seis áreas (exceção emprego). A distribuição mostra dois grupos distintos. Quase metade da amostra concentrou-se no nível mais baixo de gravidade (-4), sugerindo serem indivíduos que não apresentam problemas relacionados ao uso de álcool. Outra parcela da amostra foi distribuída entre os níveis -2 e 1, porém com maior concentração entre os níveis -2 e 0, apresentando problemas em relação ao uso de álcool. De acordo com a interpretação dos níveis de gravidade, no nível -1, por exemplo, o indivíduo relata ter bebido em mais do que um dia nos últimos 30 dias, ter bebido com certa frequência nos últimos seis meses e ter bebido regularmente por mais de um ano na vida. Além disso, o indivíduo demonstra preocupação com problemas com álcool, sente necessidade de tratamento para esses problemas e considera importante alcançar/manter a abstinência nos últimos 30 dias. 

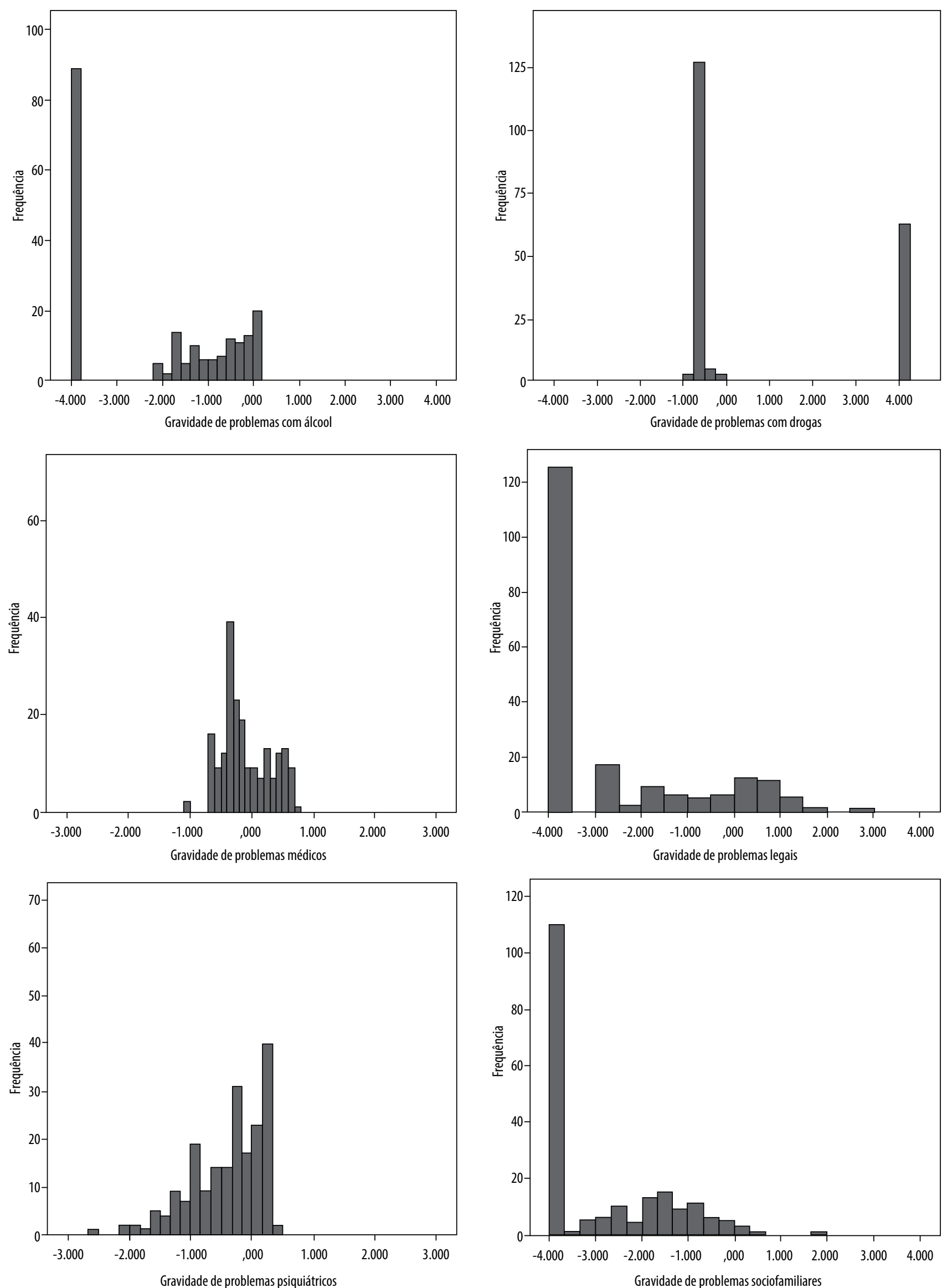

Figura 1. Distribuição de frequência dos entrevistados nos níveis de gravidade de problemas em seis áreas com base nos novos escores do ASI-6 Light. 
Em relação à área "drogas", a maioria da amostra está concentrada no nível -1. Pelos itens que compõem esse nível, pode-se interpretar que o indivíduo com escores que variam entre -1 e 0: já fez uso regular de qualquer droga por pelo menos um ano, especificamente maconha e cocaína/ crack, na vida; fez uso frequente de drogas ilícitas nos últimos seis meses; gastou dinheiro para comprar drogas nos últimos 30 dias; sentiu-se preocupado com problemas relacionados a drogas; sentiu necessidade de tratamento para esses problemas e considerou importante alcançar ou manter a abstinência total de drogas nos últimos 30 dias. Entretanto, há uma considerável parcela da amostra que está claramente concentrada no nível 4, sendo esse o nível mais alto de gravidade.

Em relação à validade concorrente das áreas "álcool" e "drogas" (Tabela 1), estabelecida pelas correlações com os escores para substâncias do ASSIST, observou-se que a área "álcool" do ASI-6 Light obteve alta correlação com o escore álcool do ASSIST ( $r=0,79)$, correlações moderadas com tabaco $(r=0,47)$ e cocaína/crack $(r=0,44)$ e baixa $(r=0,39)$ com maconha. Ao correlacionarem-se os escores do ASSIST e os escores da área "drogas" do ASI-6 Light, obteve-se alta correlação com cocaína/crack $(r=0,85)$, correlações moderadas com tabaco $(r=0,57)$ e maconha $(r=0,68)$ e baixa $(r=0,29)$ com álcool. Essas correlações foram consideradas boas, indicando a validade concorrente das áreas "álcool" e "drogas" do ASI-6 Light.

Tabela 1. Coeficientes de correlação de Pearson entre os escores de cada área do ASI-6 Light e os escores do ASSIST

\begin{tabular}{|c|c|c|c|c|c|c|}
\hline \multicolumn{7}{|c|}{$\begin{array}{l}\text { Coeficientes de correlação de Pearson entre os escores em cada área do ASI } 6 \text { Light e os } \\
\text { escores do ASSIST }\end{array}$} \\
\hline \multicolumn{7}{|c|}{ Escores das áreas ASI 6 Light } \\
\hline $\begin{array}{l}\text { Escores } \\
\text { ASSIST }\end{array}$ & Álcool & Drogas & Médica & Psiquiátrica & Legal & Família \\
\hline Tabaco & $0,47^{* *}$ & $0,57^{* *}$ & $0,20^{* *}$ & $-25^{* *}$ & $0,53^{* *}$ & $0,30^{* *}$ \\
\hline Álcool & $0,79^{* *}$ & $0,29 * *$ & $0,20^{* *}$ & $-0,09$ & $0,39^{* *}$ & $0,28 * *$ \\
\hline Maconha & $0,39 * *$ & $0,68^{* *}$ & $0,15^{*}$ & $-0,13$ & $0,58^{* *}$ & $0,31^{* *}$ \\
\hline Cocaína/crack & 0,44 & $0,85^{* *}$ & $0,17^{*}$ & $-0,11$ & $0,79^{* *}$ & $0,46^{* *}$ \\
\hline Estimulante & $0,17^{*}$ & $0,17^{*}$ & 0,06 & $-0,11$ & 0,11 & 0,05 \\
\hline Inalantes & $0,15^{*}$ & $0,18^{*}$ & 0,03 & 0,01 & $0,16^{*}$ & 0,10 \\
\hline Hipnóticos & $0,14^{*}$ & 0,10 & 0,12 & $-0,12$ & 0,13 & 0,02 \\
\hline Alucinógenos & 0,11 & $0,14^{*}$ & $-0,02$ & $-0,12$ & 0,07 & 0,01 \\
\hline
\end{tabular}

Além das áreas "álcool" e "drogas", também foram calculadas as correlações entre os escores do ASSIST e os escores das diversas áreas do ASI-6 Light. A área "legal" obteve correlação alta com cocaína/crack $(r=0,79)$, moderada com tabaco $(r=0,53)$ e maconha $(r=0,58)$ e fraca com álcool $(r=0,39)$ e demais substâncias. A área "sociofamiliar" correlacionou-se moderadamente com cocaína/crack $(r=0,46)$.
Na figura 2, encontram-se as curvas ROC das áreas "álcool" e "drogas". A ASC na área "álcool" foi de 0,93 (95\% IC: 0,88$0,96, p<0,0001$ ), indicando boa capacidade discriminatória, com alta porcentagem de classificação correta dos casos. Para sensibilidade de $80 \%$ (0,8, próximo ao ponto de inflexão da curva), obtém-se especificidade de aproximadamente $90 \%(0,9)$, apontando para boas propriedades de discriminação dos grupos da escala.

Na área "drogas", também foi observado bom equilíbrio entre especificidade e sensibilidade; a ASC foi de 0,88 (95\% IC: $0,83-0,94, p<0,0001)$. Nesse caso, a discriminação é quase perfeita, e a sensibilidade de $80 \%$ fornece especificidade de $100 \%$, ou seja, não se classificaram falsos-negativos com essa escala.
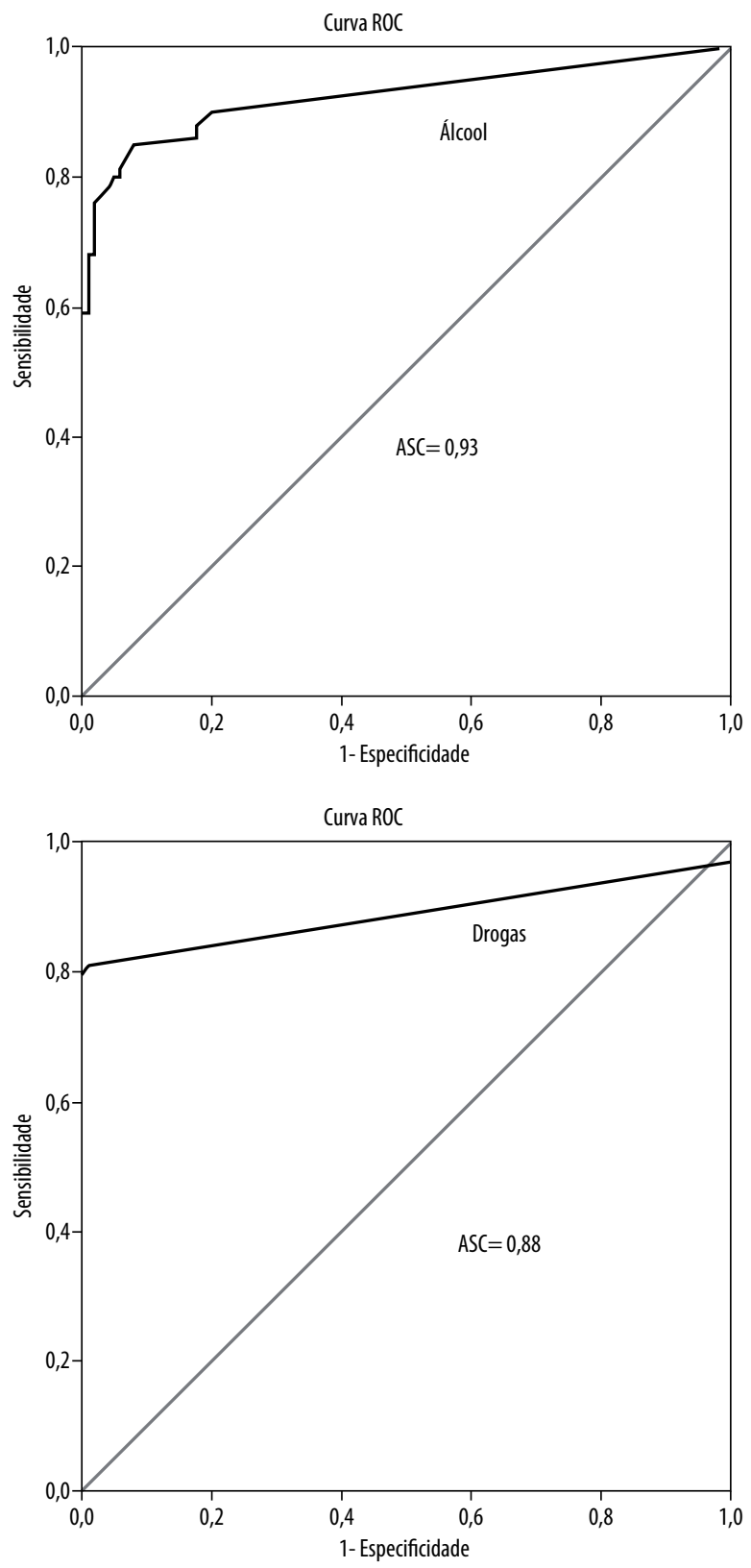

Figura 2. Curvas ROC utilizando novos escores das áreas "álcool" e "drogas" do ASI-6 Light e ASSIST. 


\section{DISCUSSÃO}

Por meio dos dados do presente estudo, foi possível verificar que a versão brasileira reduzida do ASI-6 Light apresentou boas propriedades psicométricas, o que sugere que o ASI-6 Light pode ser um instrumento útil para avaliação do uso de substâncias psicoativas e problemas relacionados. Esse instrumento tornou-se mais prático e de fácil manejo, com redução significativa do tempo de aplicação.

Em relação à validade concorrente, as áreas "álcool" e "drogas" obtiveram altas correlações com as áreas "álcool" e "drogas" do ASSIST, respectivamente. Além disso, essas áreas mostraram boa capacidade de discriminação, com altos índices de especificidade e sensibilidade, sugerindo que podem ser boas medidas para diferenciar usuários de não usuários de álcool e outras drogas. Não foram utilizados os pontos de corte gerados a partir da curva ROC, pelo fato de eles já terem sido gerados pela TRI proposto por Sartes ${ }^{15}$.

Os escores gerados para a versão reduzida possibilitam uma interpretação da gravidade de problemas em cada área de forma individualizada e inédita. O indivíduo é posicionado na escala de gravidade pelo seu escore em cada área, a partir do qual é possível identificar as características que descrevem a gravidade do paciente. Nas áreas "álcool" e "drogas", a escala de gravidade fornece uma visão de continnum de gravidade semelhante à proposta no DSM-V. Na nova versão não há mais a dicotomia entre as categorias dependência química e uso abusivo de substâncias, substituída por uma categoria unificada, com a designação de "perturbação de uso de substância", uma perturbação com gravidade clínica variável. O novo diagnóstico baseia-se em 11 critérios, fornecendo a intensidade da gravidade do problema, podendo ser moderada ou acentuada ${ }^{22}$. Vale ressaltar que os itens do ASI-6 Light que melhor discriminam indivíduos com problemas relacionados ao uso de álcool e outras drogas estão de acordo com esses critérios de diagnósticos do DSM-V, inclusive com o novo critério de presença de fissura (craving). No entanto, são necessários estudos de comparação entre os ASI-6 Light e instrumentos de diagnóstico do DSM-V para avaliação da validação concorrente.

As outras áreas fornecem uma visão de gravidade raramente encontrada em outros instrumentos. O escore em cada área permite ao clínico, por um lado, pensar em um planejamento terapêutico adequado, considerando os diferentes níveis em cada área, assim como ocorria na versão original. No entanto, os escores dessa versão não são subjetivos. Por essa mesma razão, os escores podem ser úteis aos pesquisadores que buscam uma avaliação de gravidade de problemas nas seis áreas avaliadas que apresentam um escore, mas encontram limitação quanto ao uso dos escores de gravidade subjetivos propostos pelo ASI-6 original. Vale destacar que, embora o ASI-6 tradicional utilize os escores de gravidade subjetivos, um estudo realizado por Cacciola et al. ${ }^{23}$ descreveu a derivação dos Escores Sumários Recentes (ESS) do ASI-6 tradicional utilizando a TRI não paramétrica e análises fatoriais confirmatórias. $\mathrm{O}$ estudo encontrou fortes evidências de validade dos escores e fracas para as áreas "legal", "sociofamiliar" e sua subárea "problemas com crianças". Além disso, análises de intercorrelação entre os novos escores mostraram a multidimensionalidade do ASI-6, já encontrada em outras versões ${ }^{13}$.

Embora a TRI seja um bom método para avaliar as propriedades psicométricas e gerar escores interpretáveis, uma limitação de sua utilização é que, por meio do modelo paramétrico de dois parâmetros, os escores não são facilmente gerados como em uma soma ou contagem. Assim, é necessária a utilização de recursos computacionais para a geração dos escores, conforme proposto neste estudo. Esse dado pode dificultar a utilização da versão reduzida para ambientes clínicos.

Ainda com relação aos escores, a alteração no número de categorias de cinco para quatro não alterou significativamente o posicionamento dos itens nos diferentes níveis de gravidade nas áreas. A redução de categorias otimizou a pergunta e facilitou a resposta do entrevistado. Exceção ocorreu na área "sociofamiliar", o que pode indicar a fragilidade de seu construto. Vale destacar que um dos pressupostos do modelo paramétrico de dois parâmetros da TRI é o da independência local, o que significa que a alteração em um item não deve alterar os demais ${ }^{24,25}$. No entanto, não foi o que ocorreu na área "sociofamiliar", já que a redução do número de categorias fez com que vários itens, inclusive os que não sofreram alteração, mudassem de nível de gravidade. A área "sociofamiliar" tem demonstrado fragilidade em diversos estudos das versões anteriores do ASI13,23. Embora tenha sido alterada para a sexta versão, a avaliação de seu construto com os resultados do ASI-6 Light deve ser vista com cautela.

Este estudo apresentou como limitações a avaliação de validade concorrente somente de duas áreas e utilizou amostra não representativa do país. Estudos futuros podem utilizar amostras representativas para realizar a validação concorrente das áreas "álcool" e "drogas", utilizando o DSM-V ou o ASI-6 versão tradicional como critério, e avaliar a validação concorrente das outras áreas e a confiabilidade teste-reste.

\section{CONCLUSÃO}

Com este estudo, foi possível desenvolver e avaliar as propriedades psicométricas da versão reduzida do ASI-6 Light. Boas evidências de validade concorrente da área "álcool" e "drogas" foram apresentadas, o que sugere que são boas medidas para diferenciar usuários de não usuários de álcool e outras drogas. 
Essa nova versão tornou-se um instrumento de fácil manejo e de rápida aplicação, contendo os itens que melhor avaliam o construto de gravidade de problemas com substâncias e dos problemas associados nas diversas áreas.

Foram propostos escores individuais que representam a gravidade de problemas em cada área, auxiliando o clínico a pensar em um planejamento terapêutico adequado para cada indivíduo. Tais escores não são subjetivos, o que confere resultados menos enviesados para a utilização em pesquisa.

\section{CONTRIBUIÇÕES INDIVIDUAIS}

Lídia Reis Fernandes - Trabalhou na concepção, planejamento, coleta de dados, análise e interpretação dos dados, na redação do artigo e em sua revisão crítica.

Laisa Marcorela Andreoli Sartes - Foi responsável pela supervisão de todas as etapas do estudo, participando da concepção e desenho, análise e interpretação dos dados, elaboração do artigo, revisão crítica de conteúdo intelectual e aprovação da versão final a ser publicada.

Fernando Antonio Basile Colugnati - Foi responsável pelas estimativas dos escores da TRI e revisão crítica do artigo, principalmente no que concerne aos aspectos metodológicos.

\section{CONFLITOS DE INTERESSE}

Os autores não possuem conflitos de interesse a serem declarados.

\section{AGRADECIMENTOS}

Ao Programa de Aperfeiçoamento de Pessoal de Nível Superior (Capes) e à UFJF, pela Bolsa de Iniciação Científica (BIC/ UFJF) 2013/2014.

\section{REFERÊNCIAS}

1. Mendes IAC, Luis MAV. Uso de substâncias psicoativas, um novo velho desafio. Rev LatinoAm. Enfermagem. 2004;2:299-300.

2. Formigoni ML, Castel S. Escalas de avaliação de dependência de drogas: aspectos gerais. Rev Bras Psiquiatr. 1999;26(1):5-31.

3. McLellan AT, Luborsky L, Cacciola J, Griffith J, Evans F, Barr HL, et al. New data from the Addiction Severity Index. Reliability and validity in three centers. J Nerv Ment Dis. 1985;173(7):412-23.
4. DeJong CA, Willems JC, Schippers GM, Hendriks VM. The Addiction Severity Index: reliability and validity in a Dutch alcoholic population. Int J Addict. 1995;30(5):605-16.

5. Krenz S, Dieckmann S, Favrat B, Spagnoli J, Leutwyler J, Schnyder C, et al. French version of the addiction severity index (5th Edition): validity and reliability among Swiss opiatedependent patients. French validation of the Addiction Severity Index. Eur Addict Res. 2004;10(4):173-9.

6. Liang T, Liu EW, Zhong H, Wang B, Shen LM, Wu ZL. Reliability and validity of addiction severity index in drug users with methadone maintenance treatment in Guizhou province, China. Biomed Environ Sci. 2008;21(4):308-13.

7. Luo W, Wu Z, Wei X. Reliability and validity of the Chinese version of the addiction severity index. J Acquir Immune Defic Syndr. 2010;53 Suppl 1:S121-5.

8. McLellan AT, Cacciola JC, Alterman Al, Rikoon SH, Carise D. The Addiction Severity Index at 25: origins, contributions and transitions. Am J Addict. 2006;15(2):113-24.

9. Sandí-Esquivel LE, Ávila-Corrales K. Validity of the Addiction Severity Index (adapted version) in a Costa Rican population group. Bull PAH0. 1990;24:70-6.

10. Claus RE, Kindleberger LR, Dugan MC. Predictors of attrition in a longitudinal study of substance abusers. J Psychoactive Drugs. 2002;34(1):69-74.

11. Henrique IFS, De Micheli D, Lacerda RB, Lacerda LA, Formigoni MLOS. Validação da versão brasileira do teste de triagem do envolvimento com álcool, cigarro e outras substâncias. Rev Assoc Méd Bras. 2004;50(2):199-206.

12. Kessler F, Cacciola J, Alterman A, Faller S, Souza-Formigoni ML, Cruz MS, et al. Psychometric properties of the sixth version of the Addiction Severity Index (ASI-6) in Brazil. Rev Bras Psiquiatr. 2012;34:24-33.

13. Mäkelä K. Studies of the reliability and validity of the Addiction Severity Index. Addiction. 2004;99(4):398-410.

14. Alterman Al, Cacciola JS, Habing B, Lynch KG. Addiction Severity Index Recent and Lifetime summary indexes based on nonparametric item response theory methods. Psychol Assess. 2007;19(1):119-32.

15. Sartes LMA. Propriedades psicométricas da versão brasileira do Addiction Severity Index 6 (ASI 6): uma abordagem pela Teoria de Resposta ao Item [tese]. São Paulo, SP: Universidade Federal de São Paulo; 2010.

16. Kessler F, Faller S, Souza-Formigoni MLO, Cruz MS, Brasiliano S, Stolf AR, et al. Avaliação multidimensional do usuário de drogas e a Escala de Gravidade de Dependência. Rev Psiquiatr Rio Gd Sul. 2010;32(2):48-56.

17. Hameniuk R, Poznyak V. ASSIST, The Alcohol, Smoking and Substance Involvement Screening Test: Guidelines for Use in Primary Care (Draf Version 1.1); 2008.

18. Samejima FA. Estimation of latent ability using a response pattern of graded scores. Psychometric Monograph. 1969;(17).

19. Lord FM. Applications of item response theory to practical testing problems. Hillsdale, NJ: Lawrence Erlbaum; 1980.

20. Magis D, Raiche $G$. Random generation of response patterns under computerized adaptive testing with the R Package catR. J Stat Softw. 2012;48(8):1-31.

21. Muraki E, Bock RD. Parscale: IRT based test scoring and item analysis for graded openended exercises and performance tasks. Chicago: Scientific Software, Inc.; 1997.

22. Fernandes S, Leite E, Vieira F, Santos JC. 0 anunciado DSM-5: que implicações em psiquiatria forense? Acta Méd Port. 2014;27(1):126-34.

23. Cacciola JS, Alterman Al, Habing B, McLellan AT. Recent status scores for version 6 of the Addiction Severity Index (ASI-6). Addiction. 2011;106(9):1588-602.

24. Andrade DF, Tavares HR, Valle RC. Teoria de Resposta ao Item: conceitos e aplicações. São Paulo: ABE; 2000.

25. Embretson SE, Reise SP. Item Response Theory for Psychologists. Mahwah, NJ: Lawrence Erlbaum Associates; 2000. 